\title{
Editorial: Special issue on marine safety and Rice's formula
}

\author{
Holger Rootzén · Ross Leadbetter
}

Published online: 25 March 2011

(C) Springer Science+Business Media, LLC 2011

Marine design and marine safety assurance is developing rapidly because of changes in usage and development of measurement technology. Ships now sail, or will sail, in new and challenging routes such as the north-west passage. Oil platforms are placed in ever deeper and harsher seas. The number of buoys which measure waves is increasing and is being complemented by rapidly increasing and improved satellite measurements.

Extremely high waves may destabilize ships and damage or destroy oil rigs. The danger is not determined by wave height alone, but by the entire shape of an extreme wave and its relationship to vessel geometry and motion. Further, the cyclic loading caused by recurring storms causes cumulative damage which ultimately may lead to structural breakdown. Such catastrophes are influenced by randomness in many ways. The resistances of ships are affected by randomness in material properties, and by construction work quality and the wear and tear which depends on many different random effects. Similarly, storm and wave properties are best described by random models, with extremes at the center of interest. Thus, extreme value methods, both probabilistic and statistical, are a natural and important ingredient of marine safety assurance. This provides a vast, exciting, and wide open field for research in extreme value and related risk theory.

Rice's Formula is one of the basic tools for this research, providing means for computation of expectations and higher order moments of the number of upcrossings of a high level in a random field. The first version of the formula was derived by S.O. Rice in 1944 for wave modeling, and independently in 1943 by M. Kac in the

\footnotetext{
H. Rootzén $(\varangle)$

Mathematical Sciences, Chalmers University of Technology and University of Gothenburg, 41296 Gothenburg, Sweden

e-mail: hrootzen@chalmers.se

R. Leadbetter

Department of Statistics, University of North Carolina, Chapel Hill, NC 27599-3260, USA

e-mail: mrl@email.unc.edu
} 
context of zeros of random polynomials. It has subsequently been refined, extended and used in many different contexts; see e.g. the recent (2009) monograph by Azais and Wshebor.

In this issue, the papers by Baxevani et al., and by Åberg and Podgorski develop new models for random seas and apply Rice's formula to the models, with resulting theoretical as well as practical implications. The main aims are to describe the dynamic evolution of ocean wave fields in space and time, and to go beyond the standard symmetric Gaussian models. The Laplace distribution is used for the latter purposes. The paper by Galtier uses saddle-point approximation to find computationally tractable forms of Rice's formula for a Laplace moving average process, which is one of the processes studied by Åberg and Podgorski. The paper by Anderson et al. concerns estimation of the distributions of extreme wave heights, treating both buoy and satellite data. Both data types are "sparse", for buoys because relatively few buoys are available, and those are scattered over vast areas and for satellite data because satellite passages are intermittent in time. In the paper sparseness is dealt with by introducing suitable model assumptions.

We hope that the importance and challenge of these problems will attract many more researchers to the area and, in particular, that this issue of Extremes will be a stimulus for future research.

\title{
Dynamically evolving Gaussian spatial fields
}

Anastassia Baxevani • Krzysztof Podgórski1 • Igor Rychlik

\begin{abstract}
We discuss general non-stationary spatio-temporal surfaces that involve dynamics governed by velocity fields. The approach formalizes and expands previously used models in analysis of satellite data of significant wave heights. We start with homogeneous spatial fields. By applying an extension of the standard moving average construction we obtain models which are stationary in time. The resulting surface changes with time but is dynamically inactive since its velocities, when sampled across the field, have distributions centered at zero. We introduce a dynamical evolution to such a field by composing it with a dynamical flow governed by a given velocity field. This leads to non-stationary models. The models are extensions of the earlier discretized autoregressive models which account for a local velocity of traveling surface. We demonstrate that for such a surface its dynamics is a combination of dynamics introduced by the flow and the dynamics resulting from the covariance structure of the underlying stochastic field. We extend this approach to fields that are only locally stationary and have their parameters varying over a larger spatio-temporal horizon.
\end{abstract}

\section{Estimation of return values for significant wave height from satellite data}

Igor Rychlik • Jesper Rydén. Clive W. Anderson •

Abstract Estimation of extreme wave height across the oceans is important for marine safety and design, but is hampered by lack of data. Buoy and platform data 
are geographically limited, and though satellite observations offer global coverage, they suffer from temporal sparsity and intermittency, making application of standard methods of extreme value estimation problematical. A possible strategy in the face of such difficulty is to use extra model assumptions to compensate for lack of data. In this spirit we report initial exploration of an approach to estimation of extreme wave heights using crossing methods based on a log-Gaussian model. The suggested procedure can utilize either intermittent satellite data or regular time series data such as that obtained from a buoy, and it is adapted to seasonal variation in the wave height climate. The paper outlines derivation of the method and illustrates its application to data from the Atlantic and Pacific oceans. A numerical comparison is made with the results of an annual maximum analysis for sites at which both satellite and buoy data are available. The paper concludes with a discussion of the applicability of the new approach, its relationship to other extreme value methods and desirable directions for further development.

\title{
A class of non-Gaussian second order random fields
}

\section{Sofia Åberg • Krzysztof Podgórski}

\begin{abstract}
Non-Gaussian stochastic fields are introduced by means of integrals with respect to independently scattered stochastic measures that have generalized Laplace distributions. In particular, we discuss stationary second order random fields that, as opposed to their Gaussian counterpart have a possibility of accounting for asymmetry and heavier tails. In addition to this greater flexibility the models discussed continue to share most spectral properties with Gaussian processes. Their statistical distributions at crossing levels are computed numerically via the generalized Rice formula. The potential for stochastic modeling of real life phenomena that deviate from the Gaussian paradigm is exemplified by a stochastic field model with Matérn covariances.
\end{abstract}

\section{Note on the estimation of crossing intensity for Laplace moving average}

Thomas Galtier

\begin{abstract}
In this note the upcrossing intensity of a level $u$ by a Non-Gaussian process - the Laplace Moving Average (LMA) — is estimated by means of a saddlepoint approximation of Rice's formula. The LMA-process is defined by power spectral density, skewness and kurtosis parameters. The method is illustrated by measurements of sea surface elevation and stresses in a vessel.
\end{abstract}

